\title{
Ionization of $\mathbf{H}_{\mathbf{2}}$ Rydberg molecules at a metal surface
}

\author{
G. R. Lloyd, S. R. Procter, E. A. McCormack, and T. P. Softley \\ Chemistry Research Laboratory, Department of Chemistry, University of Oxford, Mansfield Road, \\ Oxford OX1 3TA, United Kingdom
}

(Received 2 January 2007; accepted 22 March 2007; published online 9 May 2007)

\begin{abstract}
The ionization of a beam of $\mathrm{H}_{2}$ Rydberg molecules in collision with a metal surface (evaporated $\mathrm{Au}$ or $\mathrm{Al}$ ) is studied. The Rydberg states are excited in an ultraviolet-vacuum ultraviolet double-resonant process and are state selected with a core rotational quantum number $N^{+}=0$ or 2 and principal quantum numbers $n=17-22\left(N^{+}=2\right)$ or $n=41-45\left(N^{+}=0\right)$. It is found that the $N^{+}$ $=0$ states behave in a very similar manner to previous studies with atomic xenon Rydberg states, the distance of ionization from the surface scaling with $n^{2}$. The $N^{+}=2$ states, however, undergo a process of surface-induced rotational autoionization in which the core rotational energy transfers to the Rydberg electron. In this case the ionization distance scales approximately with $\nu_{0}^{2}$, the effective principal quantum number with respect to the adiabatic threshold. This process illustrates the close similarity between field ionization in the gas phase and the surface ionization process which is induced by the field due to image charges in the metal surface. The surface ionization rate is enhanced at certain specific values of the field, which is applied in the time interval between excitation and surface interaction. It is proposed here that these fields correspond to level crossings between the $N^{+}=0$ and $N^{+}=2$ Stark manifolds. The population of individual states of the $N^{+}=2$, $n=18$ Stark manifold in the presence of a field shows that the surface-induced rotational autoionization is more facile for the blueshifted states, whose wave function is oriented away from the surface, than for the redshifted states. The observed processes appear to show little dependence on the chemical nature of the metallic surface, but a significant change occurs when the surface roughness becomes comparable to the Rydberg orbit dimensions. () 2007 American Institute of Physics. [DOI: 10.1063/1.2730792]
\end{abstract}

\section{INTRODUCTION}

The scattering of molecular beams at solid surfaces is a mature field of research, and experimental work has been refined to the level of vibration-rotation quantum-state selectivity in the incoming and the scattered molecules. ${ }^{1}$ These studies reveal vital clues to the dynamical processes involved in adsorption and catalysis. The interaction of quantum-stateselected electronically excited molecules with a surface is much more challenging because of the short lifetimes of these species, but studies with beams of metastable electronically excited atoms [e.g., He $\left.\left(1 s 2 s^{3} S\right)\right]$ have been carried out, with the aim of performing, e.g., nanolithography ${ }^{2}$ or surface spectroscopy. ${ }^{3}$ Beam-surface collisional studies with electronically excited metastable $\mathrm{CO}$ and $\mathrm{N}_{2}$ have also been performed. ${ }^{4,5}$ In these cases the release of the electronic energy into the surface or the adsorbate may give rise to bond breaking and surface chemistry. In a recent paper we made a preliminary report on experiments which initiate a new aspect of state-selected beam-surface scattering by studying the interaction of a beam of very highly excited, long-lived $\mathrm{H}_{2}$ Rydberg molecules with a metal surface. ${ }^{6}$ In the present paper we discuss and analyze those preliminary results in greater detail as well as present new data that enhance understanding of the charge transfer (ionization) processes that occur. The close relationship between surface ionization and field ionization in the gas phase is highlighted.

The exotic properties of Rydberg atoms and molecules have been well studied in recent years through the use of high resolution laser spectroscopy and time-dependent wave packet experiments. ${ }^{7}$ The scaling of the lifetime of these species with $n^{3}$, where $n$ is the principal quantum number (or with $n^{4}-n^{5}$ in the presence of fields ${ }^{8,9}$ ) means that, at sufficiently high $n$, long-lived excited states can be populated for scattering studies. Furthermore, the large mean radius of the Rydberg orbital $\left(\langle R\rangle \sim n^{2} a_{0}\right)$ and the weak binding energy result in an extreme susceptibility of the electron distribution to external fields and collisional processes. A very large dipole moment can be created in a field leading to the possibility of using inhomogeneous electric fields to control the translational motion of the molecules; deflection, ${ }^{10-12}$ deceleration, ${ }^{11-14}$ and focusing ${ }^{15,16}$ of Rydberg beams have all been demonstrated.

The interaction of Rydberg atoms, either the rare gases or the alkali metals, with metallic surfaces and thin metallic films has been the subject of a few previous experimental and theoretical studies. ${ }^{17-28}$ It is found that as the atom approaches the surface, the Rydberg electron is subject to inhomogeneous electric fields caused by the generation of an image dipole in the metal, giving rise initially to a splitting of the atomic energy levels. ${ }^{7}$ At a sufficiently close distance of approach (approximately four times the Rydberg radius $\sim 4 n^{2} a_{0}$ ) tunneling ionization of the Rydberg electron occurs into the conduction band of the metal. In the experimental studies, not only can the mean radius of the Rydberg orbit be varied by spectroscopic selection of the principal quantum 
number, but the angular distribution of the electron density can also be controlled by selection of specific eigenstates in the presence of a field; thus, for example, the Rydberg atom can be polarized such that the high density part of the electron distribution either lies in front of the ionic core or trails behind the ionic core as the atom approaches the surface. ${ }^{26,27}$

The main technique employed so far to study the surface ionization process is to detect the ions that are produced and to measure the field required to pull the ions away from collision with the surface: the closer the ionization occurs relative to the surface, the greater the extraction field $\left(E_{\min }\right)$ required to overcome the attractive image charge force and the incoming kinetic energy, as given by the formula (in a.u.), ${ }^{22}$

$$
E_{\text {min }}\left(Z_{i}, T_{\perp}\right)=\left[\frac{1}{2 Z_{i}}+\sqrt{\frac{T_{\perp}}{Z_{i}}}\right]^{2},
$$

where $Z_{i}$ is the distance of ionization and $T_{\perp}=\frac{1}{2} m v_{\perp}^{2}$ is the kinetic energy perpendicular to the surface of the atom at the point of ionization. This equation is based on an image charge model which assumes that the total energy of an ion at a distance $Z$ from the surface and in the presence of an applied electric field $E$ is given, in a.u. by

$$
U=-\frac{1}{4 Z}-E Z+T_{\perp} .
$$

Experiments with Xe Rydberg atoms scattering at a Au(111) surface have shown that the distance at which ionization occurs is strongly dependent on the principal quantum number but, surprisingly, shows little dependence on the Rydberg electron angular distribution ${ }^{26,27}$ due to dipole reorientation effects (see Sec. III F below). The tunneling ionization probability at a given distance is also expected to be dependent on the detailed form of the long-range charge-induced surface potential (which is specific to the material), on the degree of surface roughness, ${ }^{19}$ and on the presence of adsorbates or thin films. ${ }^{20}$ The interaction of Rydberg atoms with a metal surface has been used to make direct measurements of the van der Waals force and retardation effects, ${ }^{29}$ and the deflection of atoms as a result of this force is potentially important in guiding ultracold electronically excited atoms ${ }^{30}$ and in ultracold atom-chip experiments. ${ }^{31}$

The use of Rydberg molecules as opposed to atoms, as discussed here, introduces an additional degree of complexity into this physical problem through the need, as shown here, to consider the vibration-rotation motion of the Rydberg molecule and also the possibility of bond dissociation.

\section{EXPERIMENT}

The excitation scheme for $\mathrm{H}_{2}$ Rydberg states has been discussed in detail in Ref. 12. In brief a population of $\mathrm{H}_{2}$ Rydberg states is produced in a pulsed, skimmed molecular beam using a vacuum ultraviolet (VUV)-UV doubly resonant excitation scheme. The VUV frequency is resonant with the $R(0) B{ }^{1} \Sigma_{u}^{+}\left(v^{\prime}=0\right)-X^{1} \Sigma_{g}^{+}\left(v^{\prime \prime}=0\right)$ one-photon transition of parahydrogen at an excitation energy of $90242.33 \mathrm{~cm}^{-1}$ populating only the $J^{\prime}=1$ level in the $B^{1} \Sigma_{u}^{+}\left(v^{\prime}=0\right)$ intermediate state. The UV frequency (laser 2) is resonant with the one-photon transitions from the intermediate state to the Rydberg series converging on the ionization thresholds associated with the para- $\mathrm{H}_{2}$ vibration-rotation levels, $v^{+}=0, \mathrm{~N}^{+}$ $=0$ and 2 of the $X^{2} \Sigma_{g}^{+}$ground ionic state at a total energy of approximately $124000 \mathrm{~cm}^{-1}$ above the ground state $\left[\operatorname{IE}\left(N^{+}\right.\right.$ $=0)=124417.507(10) \mathrm{cm}^{-1} \quad$ (Ref. 32); $\operatorname{IE}\left(N^{+}=2\right)$ $=124592.751 \mathrm{~cm}^{-1}$ (Ref. 33)]. The laser polarizations are mutually perpendicular, and, as shown in Ref. 12, the main series accessible [notated $\left.\left(n \ell N^{+}\right)_{J}\right]$ from the $J^{\prime}=1$ intermediate state are $(n s 2)_{2},(n d 0)_{2},(n d 2)_{1}$, and $(n d 2)_{2}$. Of these series it is principly the $(n d 2)_{1}$ states that survive predissociation on the time scale of our experiments $(10-12 \mu \mathrm{s}$ between excitation and surface interaction) although the $(n d 0)_{2}$ series is also observed above $n \sim 35$.

A schematic overview of the experimental apparatus is shown in Fig. 1. As in Ref. 12, the VUV beam $(\lambda$ $=110.81 \mathrm{~nm}$ ) is obtained by nonresonant frequency tripling of the frequency-doubled output ( $10 \mathrm{~Hz}, 6 \mathrm{~ns}, 5 \mathrm{~mJ} / \mathrm{pulse})$ of a Nd:YAG (yttrium aluminum garnet) pumped dye laser (Quanta Ray PDL3) in a $20 \mathrm{~cm}$ long cylindrical cell containing a $\mathrm{Kr} / \mathrm{Ar}$ mixture (1180 mbar/200 mbar). The VUV light intersects the molecular beam at an angle of $45^{\circ}$ and is focused using a lithium fluoride lens $(f=70 \mathrm{~mm})$. The UV beam $(\lambda=291-296 \mathrm{~nm}, 5 \mathrm{~mJ} /$ pulse $)$ is provided by a second frequency-doubled dye laser (Quanta Ray PDL3) pumped by the same Nd:YAG laser (Spectra Physics GCR 290) and is weakly focused using an $f=285 \mathrm{~mm}$ lens; its direction is perpendicular to the VUV beam and is at $135^{\circ}$ to the molecular beam direction.

The pulsed $(10 \mathrm{~Hz})$ molecular beam is formed by passing pure $\mathrm{H}_{2}$ gas at a backing pressure of 2 bars through a pulsed-value orifice (General Valve Series 9) of a diameter of $0.5 \mathrm{~mm}$, and then through a $1 \mathrm{~mm}$ diameter skimmer, located $3 \mathrm{~cm}$ from the valve orifice, into a differentially pumped chamber in which the pressure is $\sim 1 \times 10^{-6}$ mbar during the operation of the pulsed valve. The molecular beam is intersected by the laser beams at a distance of $170 \mathrm{~mm}$ from the skimmer face. As shown in Fig. 1(a), the excitation from the ground state to the Rydberg states occurs above the sample surface in a region of spatially homogeneous electric field between the metal surface and a mesh grid electrode $(81 \%$ transmitting $\mathrm{Ni}$ mesh with 90 lines/in.). The surface is oriented at an angle of $7^{\circ}$ with respect to the incident molecular beam axis and is mounted on a precision translation stage (National Aperture Inc. model MM-3M-ST), allowing microadjustment of its vertical position to a resolution of $0.124 \mu \mathrm{m}$. Typically, the vertical position is chosen so that excitation occurs $3 \mathrm{~mm}$ above the surface [see Fig. 1(a)], and the surface/extraction grid separation is $12 \mathrm{~mm}$. For pure $\mathrm{H}_{2}$ at 2 bar backing pressure we have previously measured a mean velocity of $2535 \mathrm{~ms}^{-1}$ in an identical pulsed valve/ skimmer combination. ${ }^{34}$ The average velocity perpendicular to the surface is therefore $\sim 309 \mathrm{~ms}^{-1}$, and it is expected that the central portion of the beam will collide with the surface $\sim 10 \mu$ s after passing through the laser excitation region. 
(a)

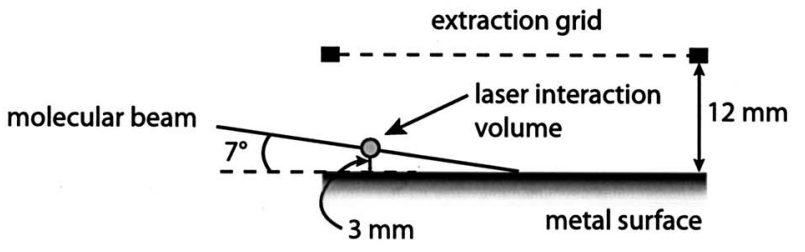

(b)

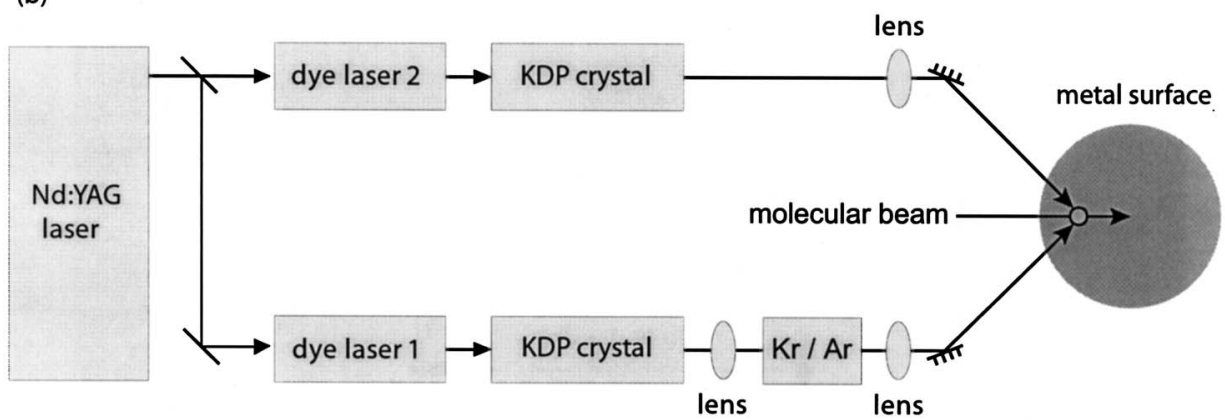

(c)

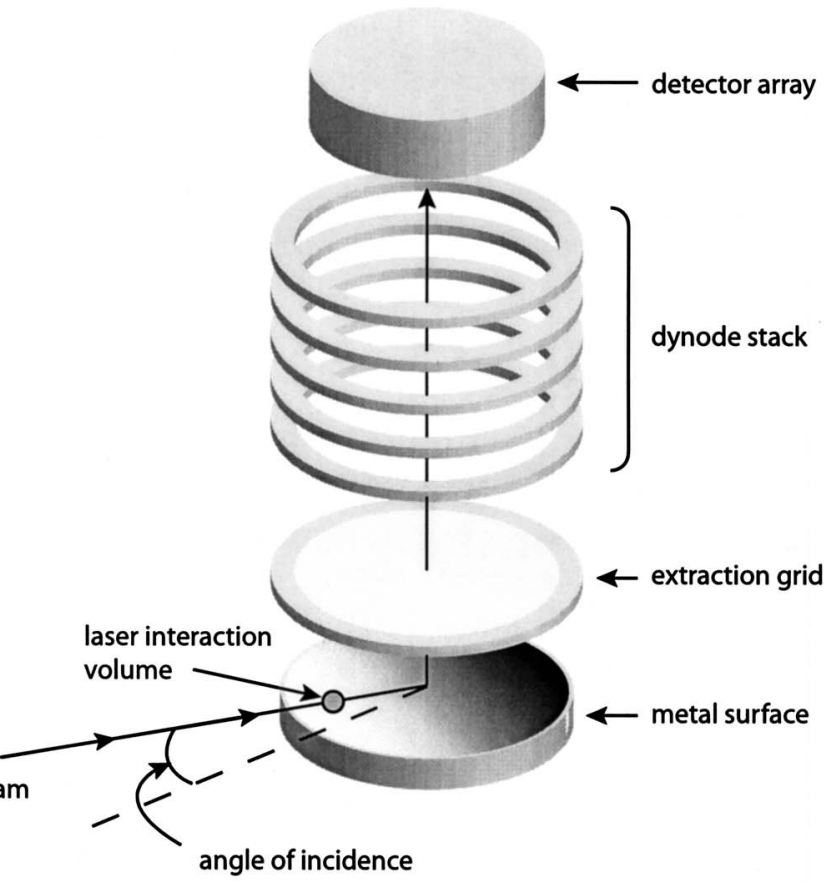

FIG. 1. Schematic diagram of the experimental apparatus: (a) Vertical profile of the interaction region; (b) horizontal profile of the interaction region, showing also the laser system; (c) detail of the extraction array.
In the majority of the experiments reported here, excitation was performed in near-zero-field conditions although a small negative bias voltage $(-10 \mathrm{~V})$ was applied to the mesh electrode to remove any ions formed directly during the excitation process. The spectrum obtained by scanning laser 2 across the range of 292.8-296.0 nm with laser 1 at a fixed wavelength is shown in Fig. 2(a). Rydberg excitation is detected through pulsed-field ionization after a delay of $800 \mathrm{~ns}$ by the application of a $+4000 \mathrm{~V}$ potential to the metal surface corresponding to a field of $\sim 3350 \mathrm{~V} \mathrm{~cm}^{-1}$ (taking the small bias field into account). The two principal series discussed above, $(n d 0)_{2}$ and $(n d 2)_{1}$, are indicated by the stick diagrams in Fig. 2(a) and converge to the $N^{+}=0$ and $N^{+}=2$ thresholds of the ionic ground state, respectively. A part of the spectrum recorded when carrying out excitation in the presence of a homogeneous field, by applying $-500 \mathrm{~V}$ to the mesh electrode, is shown in Fig. 2(b). It can be seen that the zero-field states split into hydrogenic Stark manifolds arising from the admixture of the $n d$ states into the $N^{+}=2$ high- $l$ states of the same principal quantum number $n$. $^{7,11,35}$

As discussed in the Introduction, the predominant process occurring as the Rydberg molecules approach the surface is the tunneling ionization of the Rydberg electron into the metal. The ions that are formed in this process continue to move towards the surface and are attracted to it by an oppositely charged image that is created in the metal. In order to prevent collision of these ions with the surface, a field of sufficient magnitude must be applied to pull them away. In our experiments a large, positive, variable potential up to $5000 \mathrm{~V}$ is applied to the surface $800 \mathrm{~ns}$ after excitation, 

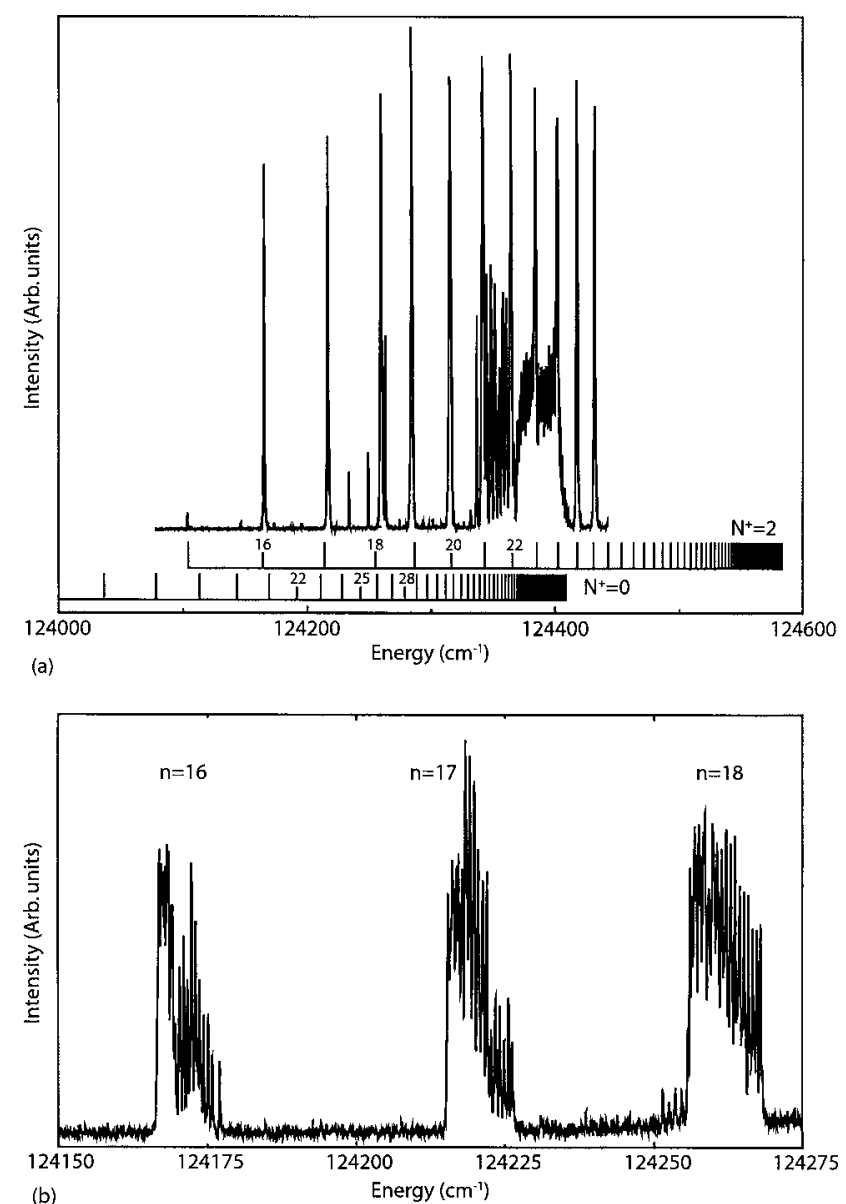

FIG. 2. (a) Zero-field excitation spectrum showing the $(n d 2)_{1}$ and $(n d 0)_{2}$ series. The spectrum is obtained by scanning laser 2 while laser 1 is tuned to the $R(0) B^{1} \Sigma_{u}^{+}\left(v^{\prime}=0\right)-X^{1} \Sigma_{g}^{+}\left(v^{\prime \prime}=0\right)$ one-photon transition of parahydrogen at an excitation energy of $90242.33 \mathrm{~cm}^{-1}$. The spectrum is shown as a function of the total $\left(1+1^{\prime}\right)$ excitation energy. (b) Reduced range of the spectrum shown in (a) with the excitation occurring in the presence of a field of $417 \mathrm{~V} \mathrm{~cm}^{-1}$.

corresponding to fields between 8 and $4125 \mathrm{~V} \mathrm{~cm}^{-1}$, in order to observe any $\mathrm{H}_{2}^{+}$ions formed at the surface. Above a certain threshold field, $E_{\text {min }}$, as determined by Eq. (1), the $\mathrm{H}_{2}^{+}$ ions are accelerated perpendicularly away from the surface through the mesh extraction electrode and travel through a field-free flight region formed by a dynode stack held at the same potential as the mesh electrode. The ion-detector assembly comprises a pair of $40 \mathrm{~mm}$ diameter multichannel plates (Burle) coupled to a phosphor screen. The fluorescence emitted by the phosphor screen can be viewed by either a photomultiplier tube (PMT) to record the total ion signal or a charge coupled device camera to obtain twodimensional information on the positions of ions hitting the detector (PHOTOLITE 3 acquisition software). The amplified output of the PMT is connected to a digital oscilloscope to record a time-of-flight spectrum.

The ion-extraction field $\left(8-4125 \mathrm{~V} \mathrm{~cm}^{-1}\right)$ is applied approximately $10 \mu$ s before the Rydberg molecules hit the surface, and therefore the ionization at the surface occurs in the presence of this field. At sufficiently high-field strengths, ions will be formed in the gas-phase region between the surface and the grid by direct field ionization or by field- induced rotational autoionization (see below). The arrival time of these ions at the detector is significantly earlier than that of the ions formed at the surface, and they also strike a different region of the detector. As a result, ions produced from either process can be easily distinguished in the timeof-flight profiles and the ion images. Examples of ion images from the camera are shown in Fig. 3(a) for the excitation of the $(20 d 2)_{1}$ state. In profile (i) the applied field is sufficiently large to ionize all Rydberg molecules in the gas phase before the interaction with the surface, whereas in (v), almost exclusively, surface ionization occurs. In (ii) to (iv) there is a partial ionization of the population by both mechanisms. The balance between these two processes, and the conditions required to facilitate them, will be discussed in more detail in Sec. III. Figure 3(b) shows the time-of-flight profiles under conditions where both surface ionization and pulsed-field ionization occur. The field ionization signal (early and sharp) moves with the delay time of application of the field, whereas the surface ionization signal (late and broad) is unchanged by the delay time, as would be expected.

In order to confirm the angle of incidence of the beam at the metal surface, a series of time-of-flight profiles was recorded in which the vertical height of the surface was varied relative to the excitation position. Data were collected for grid to surface separations in the range of $12-13.125 \mathrm{~mm}$, and two sample time-of-flight profiles are shown in Fig. 3(c). The potentials applied to the mesh grid and to the surface were varied so as to maintain a constant extraction field of $80 \mathrm{~V} \mathrm{~cm}^{-1}$. The arrival time of the ions, $T_{T}$, is given by

$$
T_{T}=T_{S}+T_{A}+T_{D}=T_{S}+\frac{\sqrt{\left(v_{i}^{2}+2 a x\right)}-v_{i}}{a}+\frac{L}{\sqrt{\left(v_{i}^{2}+2 a x\right)}},
$$

where $T_{S}$ is the time taken from excitation for the molecules to reach the point near the surface at which they are ionized, $T_{A}$ the time during which the resulting ions experience an accelerating force, and $T_{D}$ the time required for the ions to reach the detector once they have left the region between the surface and the first grid. $x$ is the separation between the surface and the grid, $L$ represents the distance from the grid to the detector $(0.197 \mathrm{~m})$ and $a$ is the acceleration due to the field. Fitting the maxima in the time-of-flight profiles to the above equation yields a value for the incidence angle of $7.0^{\circ} \pm 0.1^{\circ}$.

Three different metal surfaces were used in the experiments reported here. A deposited aluminum disk was prepared on a 3 in. polished Si (100) wafer (Compart Technologies) by metal vapor deposition in a BOC Edwards Auto 306 vacuum coater with a cryo-pumping system (giving a base pressure of $2 \times 10^{-7} \mathrm{mbar}$ ). The wafer was cleaned prior to the deposition in situ using an oxygen discharge for $10 \mathrm{~min}$. The aluminum (99.999\%, Advent Research Materials Ltd.) was then evaporated from an alumina coated molybdenum boat (R. D. Mathis, Long Beach, CA) using a $3.2 \mathrm{~V}$ and 300 A power supply. The current was controlled so that the rate of deposition on the sample was maintained at $\sim 1 \mathrm{~nm} / \mathrm{s}$, monitored using a quartz crystal microbalance (FTM5 thickness monitor, BOC Edwards) which was 


\section{Pulsed field Surface induced}

(i)

(ii)

(iii)

(iv)

(v)
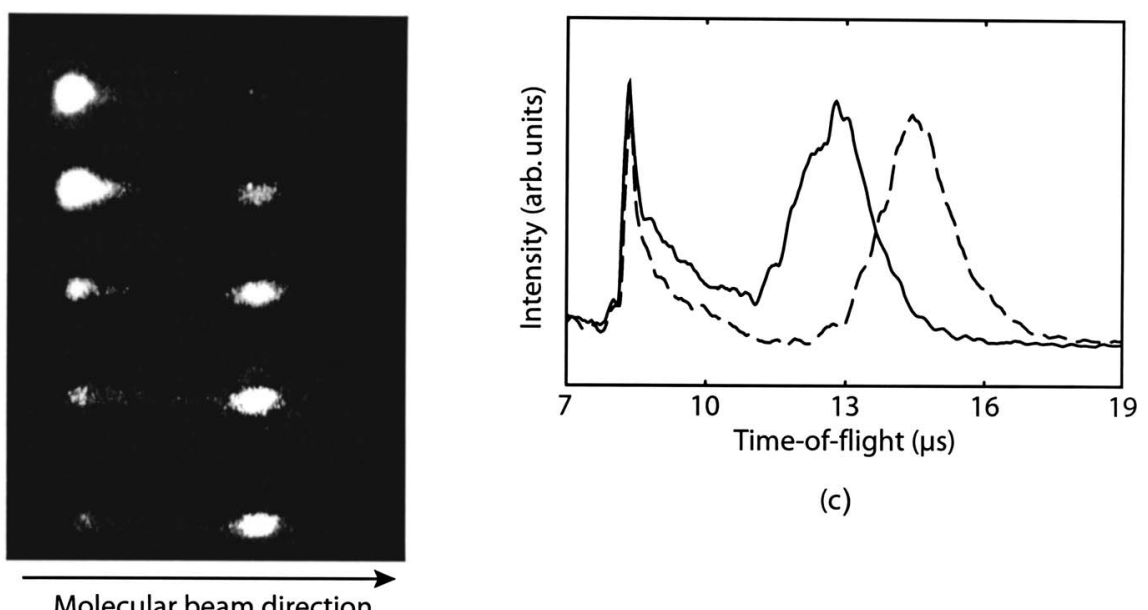

(c)

(a)

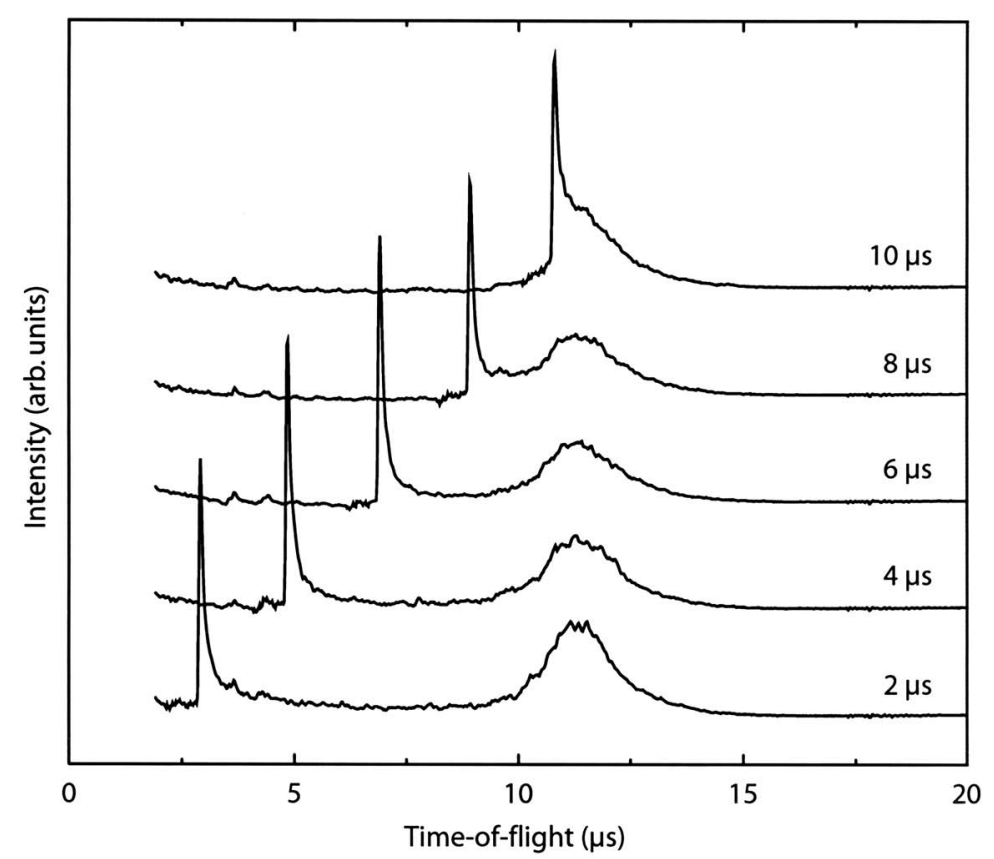

(b)

FIG. 3. (a) Ion images showing gasphase pulsed-field ionization and surface-induced ionization signals to the left and right, respectively. The $(20 d 2)_{1}$ state is populated, and the extraction fields employed are (in $\mathrm{V} \mathrm{cm}^{-1}$ ) (i) 396, (ii) 388, (iii) 379 , (iv) 371, and (v) 363. (b) Time-of-flight ion-signal profiles illustrating the effect of altering the delay of the ionextraction pulse with respect to the laser excitation pulses. (c) Dependence of the ion time-of-flight signal with the vertical height of the surface relative to the laser excitation position [surface-grid separations of $12 \mathrm{~mm}$ (normal line) and $12.8 \mathrm{~mm}$ (dashed line)]. mounted adjacent to the wafer, both $120 \mathrm{~mm}$ from the aluminum source. During the evaporation the wafer was rotated at approximately $5 \mathrm{rpm}$ and the pressure was kept below 1 $\times 10^{-6}$ mbar at all times. The evaporation was stopped once the film thickness had reached $100 \mathrm{~nm}$, and then the system was allowed to cool to room temperature before venting and removing the coated wafer. The surface topography was characterized both before and after deposition using an atomic force microscope (Digital Instruments, Multimode SPM) operated in tapping mode using a NST-NCHF-R silicon probe (Nascatec Technologies $\mathrm{GmbH}$ ) and an "E" scanner.

A deposited gold disk was prepared similarly on a 3 in. polished silicon wafer. In order to give good adhesion for the gold layer, an initial $5 \mathrm{~nm}$ thick layer of chromium was deposited onto the silicon wafer. This was sublimed from a chrome-plated tungsten rod (R. D. Mathis, Long Beach, CA) using a $10 \mathrm{~V}$ and 90 A power supply. The gold $(99.99 \%$,
Alfa) was then deposited in an identical manner to the aluminum. The surface topography was again characterized using atomic force microscopy (AFM) techniques.

An example of an AFM image recorded for the evaporated gold surface is shown in Fig. 4. It is found that the mean surface roughness $R_{a}$ for both aluminum and gold is less than $1 \mathrm{~nm}$, which is considerably less than the mean Rydberg orbit radius of the interacting molecules; the gold and aluminum surfaces can therefore be regarded as smooth as "seen" by the Rydberg molecule.

For comparison with the behavior of a rougher surface, an aluminum disk with a diameter of $50 \mathrm{~mm}$ was machined from an aluminum block and polished on a lathe. The $R_{a}$ of the disk is found to be approximately $230 \mathrm{~nm}$ but cannot be well defined due to the large, micron-sized, objects present on the surface. In any case the deviation of surface heights is significant on the scale of the molecular Rydberg radius, and 


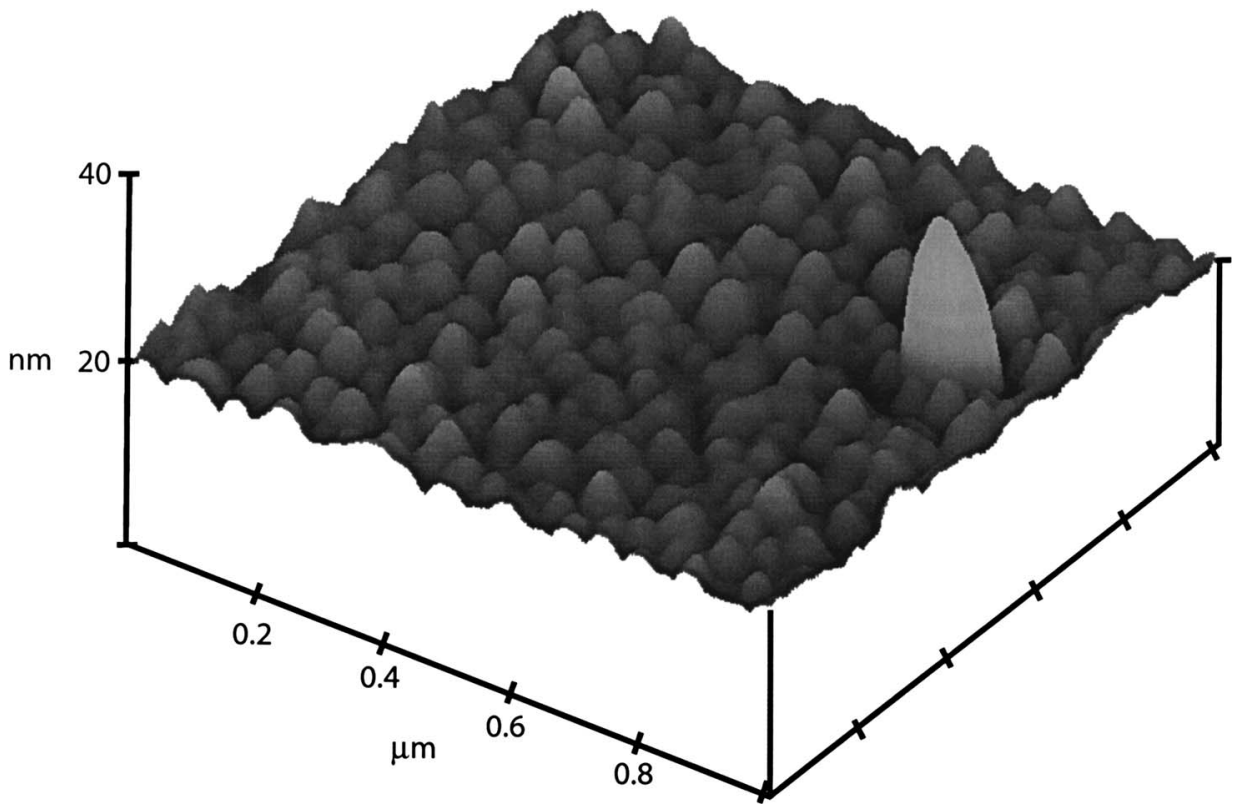

FIG. 4. Sample AFM image of the deposited gold surface.

as such this surface can be regarded as rough as seen by the Rydberg molecule.

\section{RESULTS}

\section{A. Surface ionization profiles for aluminum}

The variation in the surface ionization signal for the deposited aluminum surface recorded as a function of the applied ion-extraction field strength is shown in Fig. 5 [note the expanded horizontal scale in (a) compared to (b)]. The profiles show the behavior observed when exciting a population of $(n d 2)_{1}$ Rydberg states with $n=17-22$ as assigned in the

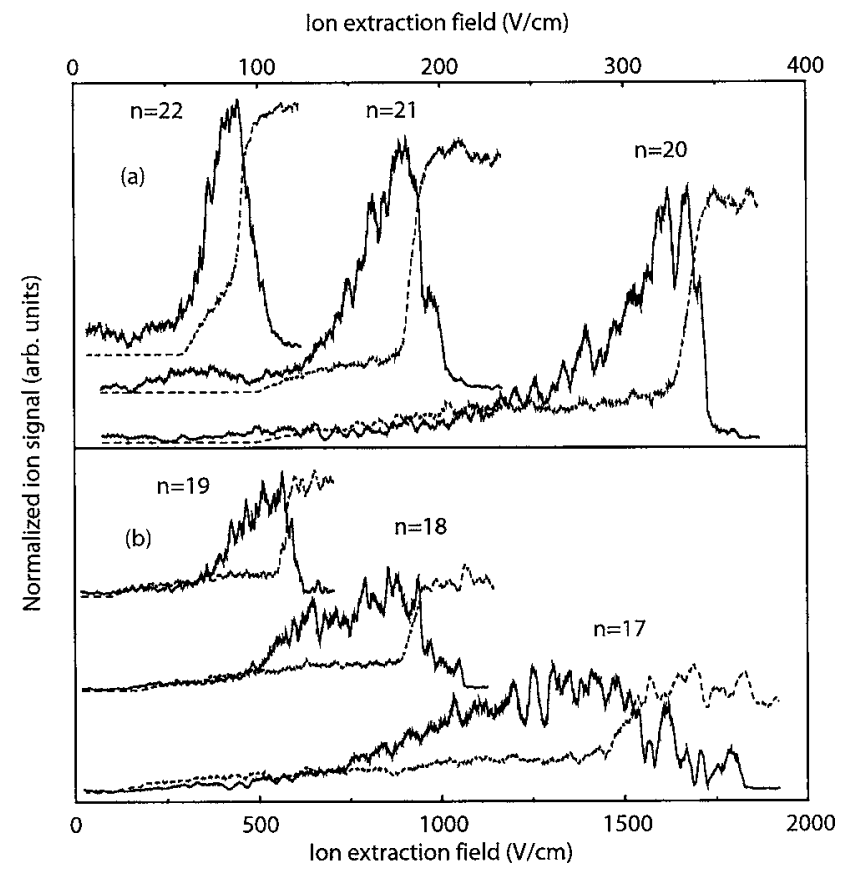

FIG. 5. Surface ionization signal (normal line) and direct field ionization signal (dashed line) vs applied extraction field for excitation of $(n d 2)_{1}$ Rydberg states incident on the deposited aluminum surface for a range of principal quantum numbers (a) $n=20-22$ and (b) $n=17-19$ [note the different horizontal scales for (a) and (b)]. figure. In all cases the ion-extraction field is pulsed on 800 ns after excitation and $\sim 10 \mu$ s before surface interaction. The Rydberg molecules fly in the presence of this field towards the target surface where ionization occurs. It is seen that in each case there is an onset of the surface ionization signal at a threshold field that decreases with increasing principal quantum number, and then there is a gradual rise of the extraction probability. It should be noted that the lack of signal at lower fields does not imply that no surface ionization occurs - only that no signal is detected because the field is not strong enough to pull the ions away from the surface. The rapid decrease in signal at the high-field end of the profiles arises because the Rydberg molecules are directly ionized in the gas phase (or undergo field-induced autoionization) at such fields prior to reaching the surface. As mentioned previously, this direct ionization occurs shortly after the extraction pulse is applied, and consequently, ions formed in this manner are not detected in the same time gate as the ions under investigation. The ion signals obtained by setting the detector time gate on the early peak in the timeof-flight spectrum, so as to detect only field ionization in the gas phase, are also shown as dashed lines in Fig. 5. It is clearly seen that this signal rises as the surface ionization signal falls.

If surface-induced ionization occurs at a molecule/ surface separation $Z_{i}$, then the minimum external field in a.u. that must be applied to prevent the ions striking the surface and hence the minimum field required for ion detection are given by Eq. (1). This equation may also be rearranged to give a value of the minimum separation from the surface at which ions formed will have their velocity reversed and be detected for a given field and kinetic energy,

$$
Z_{\text {min }}\left(E, T_{\perp}\right)=\frac{T_{\perp}+\sqrt{E}}{2 E}+\frac{1}{2} \sqrt{\frac{T_{\perp}^{2}+2 T_{\perp} \sqrt{E}}{E^{2}}} .
$$

Consequently, Eqs. (1) and (4) provide a link between the ion signal profiles recorded and the molecule/surface separation at which ionization occurs. 


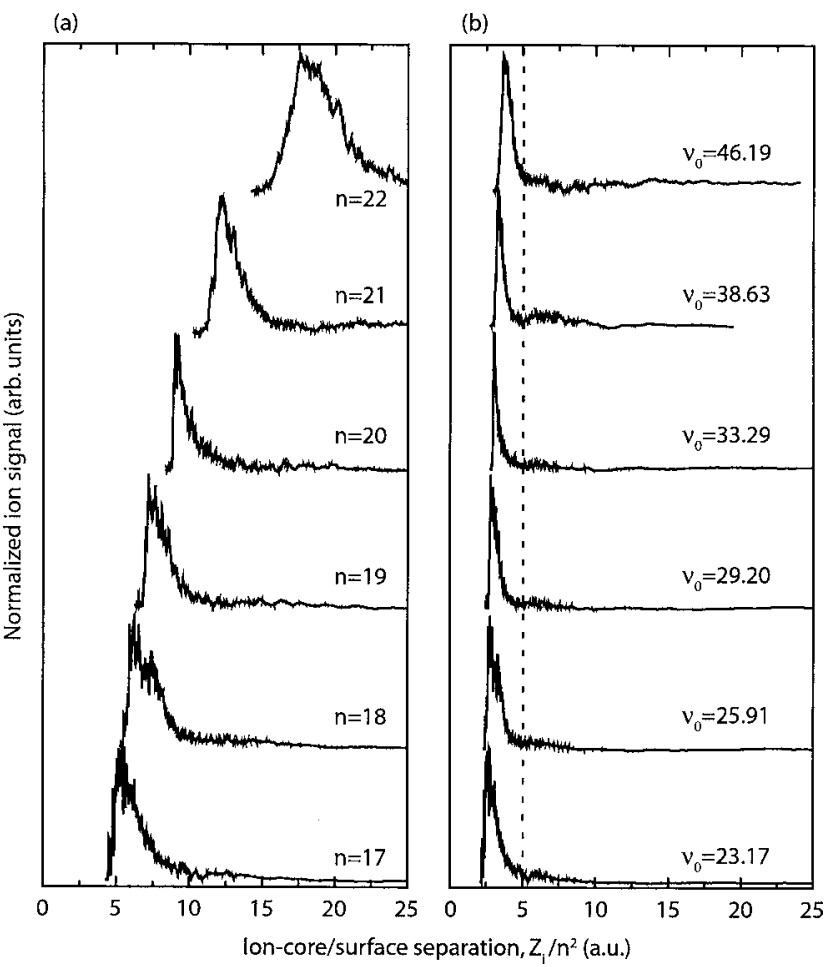

FIG. 6. (a) Surface ionization profiles for $n=17-22$ Rydberg states at an aluminum surface plotted as function of $Z_{i} / n^{2}$ using Eq. (4) and assuming a perpendicular velocity of $309 \mathrm{~ms}^{-1}$. (b) The same data are plotted as in (a) except that $\nu_{0}$, as defined in Eq. (5), replaces $n$ in the horizontal scaling.

Ionization of the Rydberg molecule requires the overlap of the Rydberg electron wave function with the metal surface. Classically, the radius of the Rydberg orbit scales with $n^{2}$, and so it is not surprising to find that in the data presented in Fig. 5, the onset of the surface ionization signal occurs at higher fields when states of lower principal quantum number are populated. This increase in the field reflects ionization occurring at a smaller molecule/surface separation. Hydrogenic theory ${ }^{22}$ and previous experiments involving xenon Rydberg states ${ }^{18}$ have found that the threshold field scales approximately as $n^{-2}$.

Using Eq. (4), the $x$ axis of Fig. 5 can be rescaled to represent the minimum distance from the surface at which the detected ions were formed. For a preliminary analysis the spread of kinetic energy in the molecular beam is ignored and a value of $T_{\perp}=3.6352 \times 10^{-5}$ a.u. is used which corresponds to the mean kinetic energy of the molecules; i.e., with $\bar{v}=2535 \mathrm{~ms}^{-1}$ and $\bar{\theta}=7.0^{\circ}$. If the $x$ axis is further scaled by a factor of $1 / n^{2}$, where $n$ is the principal quantum number of the initially populated Rydberg state, then it would be expected, assuming a hydrogenic behavior, that all the scaled profiles for different $n$ would be essentially identical. This rescaling is shown in Fig. 6(a) and it is clear that the simple scaling relationship does not apply to the $(n d 2)_{1}$ Rydberg states of $\mathrm{H}_{2}$.

In fact, the scaled profiles suggest that as $n$ increases, ionization occurs at progressively larger distances from the surface than the value of $\sim Z_{i}=4.0 n^{2} a_{0}$, as found previously for xenon atoms. For example, for $n=22$ this distance is in the range of (15-20) $n^{2} a_{0}$. If, however, an effective principal quantum number, $\nu_{0}$, calculated with respect to the $N^{+}=0$ threshold, is assigned to each state,

$$
\nu_{0}=\left[\frac{1}{n^{2}}-\frac{\left(\mathrm{IP}_{2}-\mathrm{IP}_{0}\right)}{R_{H}}\right]^{-1 / 2}
$$

(where $\mathrm{IP}_{i}$ is the ionization limit of the Rydberg series with rotational angular momentum quantum number $i$ and $R_{\mathrm{H}}$ is the Rydberg constant for $\mathrm{H}_{2}$ ), then, a satisfactory scaling relationship with $\nu_{0}^{2}$ is found, as illustrated in Fig. 6(b). The ionization occurs over a range of $(2.5-5) \nu_{0}^{2} a_{0}$.

The explanation for this behavior, as proposed in our preliminary communication, ${ }^{6}$ is that the overall ionization mechanism involves a complete transfer of rotational energy from the $\mathrm{H}_{2}^{+}$ion core to the Rydberg electron, so that the molecule behaves as if the Rydberg electron was in a higher$n$ state. This process is analogous to the phenomenon of field-induced autoionization (as discussed further in Sec. III F) which has previously been observed for the ionization of these same states in the gas phase. ${ }^{11,12}$ At a certain distance from the surface, the molecule in the $N^{+}=2$ Rydberg state finds itself at an energy (shifted by interaction with the surface) which is degenerate with the continuum of the $\mathrm{N}^{+}$ $=0$ channel, the threshold for which has been lowered by the surface interaction. Coupling between the $N^{+}=0$ and $N^{+}=2$ channels leads to autoionization. In other words, the field due to the image charges in the surface induces the forced rotational autoionization of the $N^{+}=2$ states.

In the present case the mechanism for this coupling between the $N^{+}=0$ and $N^{+}=2$ channels is not straightforward; the states populated using the perpendicular laser polarizations are of $M_{J}=0$ character, and, as shown in Ref. 12, there should be no coupling between the $N^{+}=2$ and $N^{+}=0$ states with $M_{J}=0$ even in the presence of a homogeneous field. The observed surface-induced autoionization must therefore result from a degree of $M_{J}$ mixing caused by field inhomogeneity near the surface or by the imperfect crossed polarization of the lasers. Whatever the mechanism, the time scale on which this autoionization must take place, if it is to occur before the molecule is buried into the surface, is of the order of $1 \mathrm{~ns}$. We show later that this coupling is apparently enhanced at certain applied fields (see Secs. III B and III E).

\section{B. Surface ionization behavior of the $\mathbf{N}^{+}=0$ states}

We have proposed above that the behavior displayed by the $N^{+}=2$ Rydberg states is associated with the coupling between the $N^{+}=2$ and $N^{+}=0$ channels via a mechanism akin to forced rotational autoionization. This effect is therefore a pure molecular mechanism that could not be exhibited by atomic Rydberg systems. If this explanation is correct, then one might expect that the surface ionization versus field profiles of the $N^{+}=0$ Rydberg states, which have zero rotational energy, would not be able to undergo this mechanism and that the distance of ionization would scale with $n^{2}$, as observed for atomic Rydberg states. However, there is also the possibility that a reverse coupling process with the $N^{+}=2$ states could occur such that the Rydberg electronic energy is 

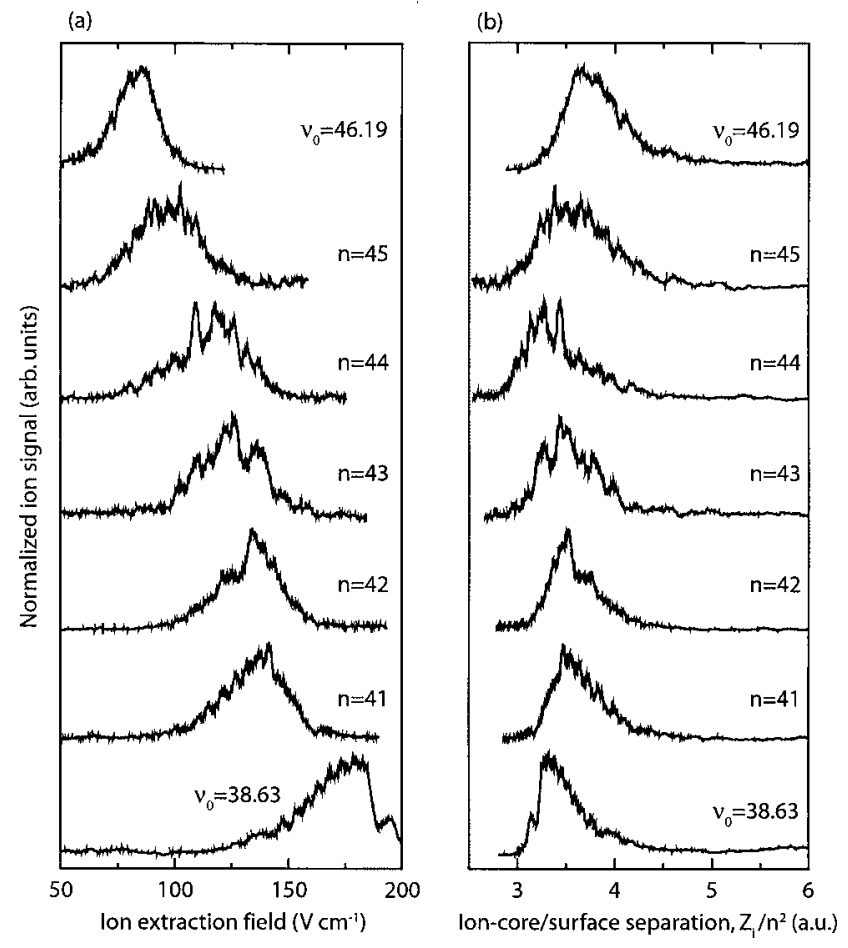

FIG. 7. Surface ionization profiles for the $n=41-45$ states belonging to the $(n d 0)_{2}$ series, together with data for the $(n d 2)_{1}, n=21 \quad\left(\nu_{0}=38.63\right)$, and $n$ $=22\left(\nu_{0}=46.19\right)$ states. (b) The same data as in (a) but plotted as a function of $Z / n^{2}$ [or $Z / \nu_{0}^{2}$ in the case of the $(n d 2)_{1}$ states].

transferred into rotation as the molecule approaches the surface, inhibiting ionization.

The $N^{+}=0$ states in the range $n=17-22$ are too short lived, because of predissociation, to be of use in the surface scattering experiments. The $(41-45 d 0)_{2}$ states are longer lived and lie energetically between the $n=22$ and $n=21$, $(n d 2)_{1}$ Rydberg states; they are clearly observed in the spectrum at around $124350 \mathrm{~cm}^{-1}$ shown in Fig. 2(a). The associated signal versus field profiles obtained by populating these states, recorded in an identical manner to the data collected for the $N^{+}=2$ states, are shown in Fig. 7(a). Once again, the profiles form a series where the extraction field required for ion detection decreases as the principal quantum number of the state initially populated increases. The variation is less marked, however, than for the $N^{+}=2$ states because the percentage increase in the mean orbital radius on going from $n \rightarrow n+1$ is smaller at higher $n$. For comparison the data for the $n=22$ and $n=21, N^{+}=2$ Rydberg states, which lie in an energetically similar region of the spectrum, are included in the same figure. These states are labeled with their effective principal quantum numbers with respect to the $N^{+}=0$ limit, $\nu_{0}$.

Once again, if the $x$ axis is rescaled using Eq. (4), such that it represents the ionization distance rather than the field, a scaling factor for the minimum ionization distance $Z_{i}$ can be determined. Such a transformation leads to data presented in Fig. 7(b). In the case of the $N^{+}=0$ states the final ionization distances have been divided by $n^{2}$ while those of the $N^{+}=2$ states have been divided by $\nu_{0}^{2}$ as outlined in the previous section. It is clear that the $N^{+}=0$ data do indeed scale as $n^{2}$ as observed for atomic Rydberg states previously, ${ }^{22}$ with surface ionization observed over the range of (3-4.5) $n^{2} a_{0}$. The comparison with the $N^{+}=2$ data lends considerable weight to the conclusion that surface ionization of Rydberg states initially prepared in $(n d 2)_{1}$ levels is mediated through a process of forced autoionization due to coupling with the $N^{+}=0$ continuum in the presence of the applied field and the image charge fields.

An unexpected feature of the data presented in Figs. 5 and 7 for both the $N^{+}=2$ and $N^{+}=0$ states is the presence of a strong resonance behavior in the probability of detecting surface ionization at specific fields. The profiles do not vary smoothly as the applied field is changed, as was apparently the case in previous measurements on atomic systems. ${ }^{22}$ This structure in the profiles is experimentally reproducible as illustrated in Fig. 4 of Ref. 6, and the origin of this structure is discussed in Sec. III E.

\section{The nature of the target surface}

All the results presented so far have involved $\mathrm{H}_{2}$ molecules incident on a deposited aluminum surface, and it is important to ask to what extent the ionization behavior of the Rydberg states is also dependent on the properties of the surface itself. The total potential experienced by the incident particle is dependent on a surface potential term ${ }^{22}$ related to the electronic properties of the surface. In order to investigate whether this has an observable impact on the signals, the experiments were repeated with an evaporated gold surface. Data were collected for the $(n d 2)_{1}$ Rydberg states for the range of principal quantum numbers $n=19-22$ using the same experimental methodology as previously used for the deposited aluminum surface. The results are presented in Fig. 8(a).

It is found that the change to gold has very little effect on the ionization characteristics of the $(n d 2)_{1}$ Rydberg states. The $N^{+}=2$ states continue to show the same surface-induced rotational autoionization behavior. The results for both aluminum and gold target surfaces are compared in Fig. 4(b) of Ref. 6 for the $\mathrm{H}_{2}(n d 2)_{1}$ states with principal quantum number $n=20$. The observed resonant structure appears to be independent of the type of metal, although there may be subtle differences which are not distinguishable within the signal-to-noise ratio.

The form of the field profile is, however, dependent on the surface roughness. Replacing the smooth surfaces prepared via metal-vapor deposition with a machined aluminum disk results in a marked change in the ionization behavior of the Rydberg states, as shown in Fig. 8(b). Comparing these data with the deposited aluminum and gold data in Figs. 5 and 8(a), respectively, it appears that ionization is detectable at much lower field strengths for the case of the rough surface, implying that ionization occurs over a greater range of molecule/surface separations. The machined aluminum has a characteristic roughness of order $230 \mathrm{~nm}$ with some micronsized defects on the surface. Such significant irregularities lead to a range of effective incident angles relative to the local orientation of the surface plane, resulting in the field profile being extended to lower (corresponding to shallower incident angles) and higher fields. The extension of the pro- 


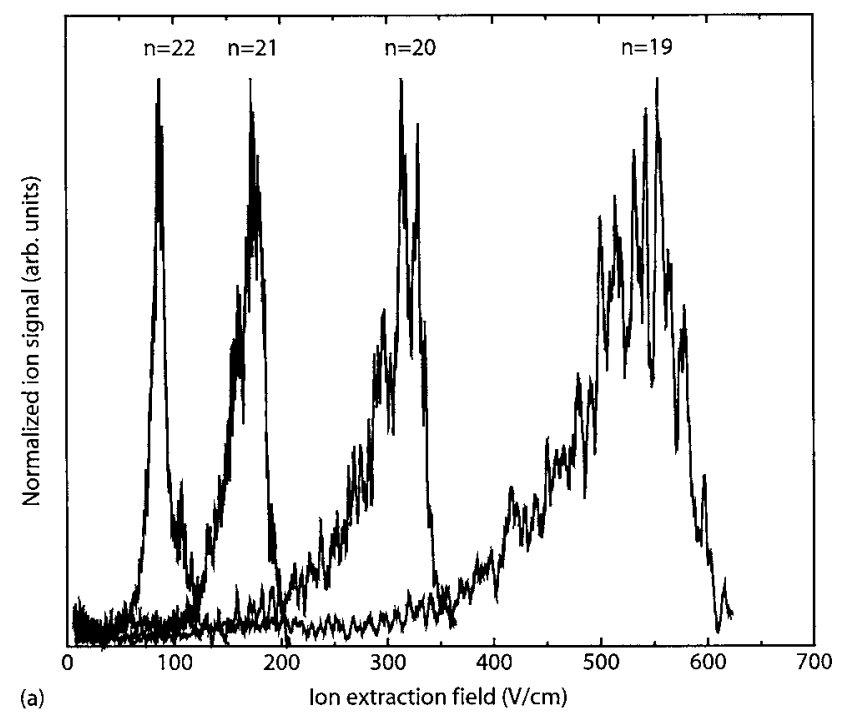

(a)

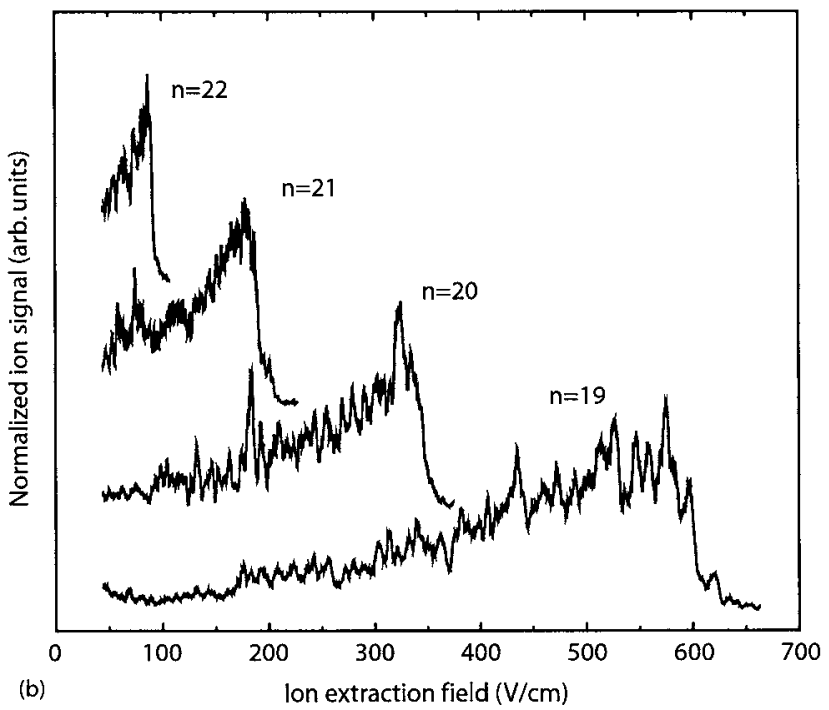

FIG. 8. (a) Surface ionization profiles for population of the $(n d 2)_{1}, n$ $=19-22$, Rydberg states incident on the deposited gold surface; (b) Similar to (a) except for the machined aluminum surface.

file to higher fields is not observable due to the fact that direct field ionization successfully competes at such fields, regardless of the nature of the surface, preventing the molecules from reaching the region in which surface ionization may occur. It may also be the case that surface irregularities cause the field to be locally inhomogeneous, further modifying the ionization characteristics of the Rydberg molecules. It is interesting to note, nevertheless, that the resonances in the field profile are still clearly observed at essentially the same fields.

\section{Simulation of surface ionization profiles for $\mathbf{N}^{+}=2$ states}

There are three principal factors contributing to the ionization profiles shown in Figs. 5-8: (a) the distribution of ionization distances from the surface, (b) the distribution of the perpendicular components of the kinetic energy $T_{\perp}$, and (c) the characteristics of the competing field ionization in the gas phase. There is potentially a fourth contributing factor, which is the redistribution of quantum states of the Rydberg molecules through black-body-radiation-induced transitions prior to interaction with the surface as well as nonadiabatic transitions as the field is switched on, but for the moment we assume that this is adequately captured in (a).

Trajectory calculations are performed to simulate the experimental results using the following model: (i) The initial velocity of molecules ejected by the pulzed nozzle is selected randomly from a velocity distribution corresponding to perpendicular and parallel temperatures both equal to $1 \mathrm{~K}$. The parallel velocity components are added to a mean beam velocity of $2535 \mathrm{~ms}^{-1}$. (ii) The molecules are ejected from the nozzle over a range of angles because of the nonzero perpendicular velocity components, but only those trajectories that pass through the skimmer and the laser focal volume (assumed to have square cross section) are retained in the simulations. (iii) The surface is oriented at $7^{\circ}$ to the central beam axis, and the kinetic energy perpendicular to the surface calculated for each trajectory. (iv) The probability of ionization in the gas phase as a function of field is assumed to increase linearly from zero at a threshold field $E_{\min }$ to one at field $E_{\max }$ where $E_{\min }$ and $E_{\max }$ scale with the squared effective principal quantum number $\nu_{0}^{-2}$ rather than the actual principal quantum number. The experimental field ionization curves shown in Fig. 5 are reasonably well fitted with

$$
E_{\min }=\frac{A}{\nu_{0}^{2}}, \quad E_{\max }=\frac{B}{\nu_{0}^{2}},
$$

with the constants $A$ and $B$ determined from the $n=18$ curve. (v) The surface ionization process is assumed to take place over a Gaussian distribution of distances from the surface; the center of the Gaussian and the width are treated as adjustable parameters and are assumed to scale with $\nu_{0}^{2}$. The field required to extract the ions is calculated using Eq. (1).

For each trajectory that is retained in step (ii) the perpendicular kinetic energy is calculated, and then a random distance of ionization is selected from the Gaussian distribution in (v). The trajectories are binned according to the minimum field required to extract the ion [using Eq. (1)] and the histogram is integrated to obtain a surface ionization versus field profile. The direct field ionization profile [Eq. (6)] is then applied to generate the high-field cutoff of the simulated profiles. The results are shown in Fig. 9, and, generally, good agreement with the experimental curves is observed provided the resonant structure is ignored. In fact, the rise of the signals is determined almost entirely by the spread of ionization distances, while the predicted spread of kinetic energies and incidence angles has a negligible effect on the overall profiles. The best fit values characterizing the distribution of ionization distances were found to have a Gaussian width equal to $1.0 \nu_{0}^{2} a_{0}$ centered at $3.1 \nu_{0}^{2} a_{0}$. The spread of ionization distances occurs because the surface-induced autoionization occurs over a finite time window, during which the motion of the Rydberg molecule is significant. The later in the time window the ionization occurs, the larger the field required to extract the ion.

It is evident, particularly from the $n=17$ profile, that the long tail at low field is not fully accounted for in the data, nor is the extension of the profile at the high-field end. Theoretical work, to be published elsewhere, shows that the tail 


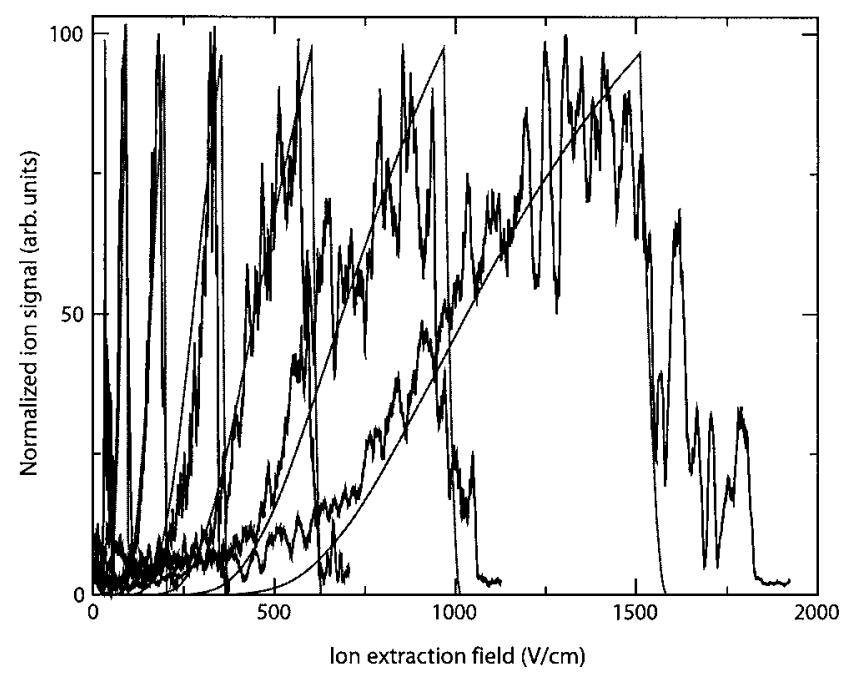

FIG. 9. Simulated surface ionization profiles, as described in the text, using a Gaussian distribution of ionization distances of center $3.1 \nu_{0}^{2} a_{0}$ and of width $1.0 \nu_{0}^{2} a_{0}$, in comparison with the experimental profiles for the deposited aluminum surface.

is due to redistribution of the Rydberg population by blackbody radiation stimulated transitions. Redistribution of population amongst $\mathrm{H}_{2}$ Rydberg states was also observed by Morgan et al., ${ }^{36}$ but under their experimental conditions they attributed the transfer to spin-orbit-induced mixing within a Stark manifold of a given principal quantum number. In the present work the data can only be explained by invoking $n$-changing processes; these are well matched to the $300 \mathrm{~K}$ black-body spectrum in the range of principal quantum numbers considered here.

\section{E. The structure in the surface ionization field profiles}

As highlighted in Sec. III B, the ion signal versus field profiles generally show resonant enhancement of the surface ionization signal at specific fields and, as discussed in Sec. III $\mathrm{C}$, the resonance behavior is independent of the type of metal used to form the surface. The origin of the observed structure must therefore lie in the evolution of the character of the Rydberg state in the period between excitation and surface interaction. There are five distinct phases in the evolution of the Rydberg state: Initially, it is prepared with the $(n d 2)_{1}$ character within the 5 ns duration of the laser pulse and will continue to be exposed to the laser fields within that $5 \mathrm{~ns}$ period, interacting with any nearby ions, electrons, and Rydberg molecules formed in the laser interaction volume, and with the small applied discrimination field $\left(\sim 7 \mathrm{~V} \mathrm{~cm}^{-1}\right)$. In the second phase there follows an $800 \mathrm{~ns}$ time span in which the Rydberg molecules are held at the constant discrimination field. In the third phase, starting $800 \mathrm{~ns}$ after laser excitation, the variable ion-extraction field is ramped up over a period of $\sim 100 \mathrm{~ns}$ to a prescribed magnitude, and then in the fourth phase the field is held constant again for several microseconds. Once the Rydberg molecules move to within a distance of approximately $50 n^{2} a_{0}$ from the surface they will start to experience the additional field due to the image charges in the surface and there will be a final fifth stage of evolution of the wave function ending in ionization. Clearly, the resonant enhancement of the surface ionization signal is associated with either the third, fourth, or fifth phases of this evolution (as it is only during these periods that the resonant field is present).

A possible explanation for the resonance enhancement is that the peaks in the surface ionization profiles correspond to the fields of level crossings between the initially populated $N^{+}=2$ states and suitable $N^{+}=0$ states. We assume that as the field is ramped up in the third phase, the system follows the initially populated level adiabatically within the $N^{+}=2$ manifold, but, owing to the very weak $N^{+}=0 / N^{+}=2$ coupling, passes diabatically through any $N^{+}=0 / N^{+}=2$ crossings during the field ramp.

Analysis of the calculated Stark map reproduced from Ref. 12 in Fig. 10 indicates that there are a number of crossings predicted in the range of $750-1500 \mathrm{~V} \mathrm{~cm}^{-1}$ between the redshifted level of the $N^{+}=2, n=17$ Stark manifold, with which the experimentally populated $(17 d 2)_{1}$ level correlates adiabatically (ignoring crossings with the $N^{+}=0$ manifolds), and the blueshifted levels of the $N^{+}=0, n=21$ and 22 Stark manifolds. Our working hypothesis is that if the ramped field leads the molecules to such a crossing, then the surface ionization probability is enhanced. In Fig. 10(c) a simulated ionization profile has been constructed by assuming that there is a Gaussian-shaped ionization probability distribution (width $50 \mathrm{~V} \mathrm{~cm}^{-1}$ ) around each of the level crossing positions marked in Fig. 10(b). The surface ionization profile for $n=17$, shown in Fig. 9, has approximately the same number of peaks in this field range. Exact agreement in peak positions would depend on the accuracy of the calculated Stark map, but the agreement in positions of prominent features is encouraging.

But the question remains as to why should holding the field at such level crossings affect the surface ionization behavior? In the case of the excitation of an $N^{+}=2$ state, it was demonstrated in Secs. III A and III B that the ionization mechanism was essentially a forced rotational autoionization process and therefore requires a coupling between the $N^{+}$ $=2$ states and the $N^{+}=0$ continuum. The time scale associated with the coupling must be fast compared to the time it takes for the molecule to move from the point where ionization is energetically possible to the point where the molecule is too close to the surface for the ion to be pulled away by the field. This time scale is less than $1 \mathrm{~ns}$ for the incident velocities employed in our experiments. If the rate of the $N^{+}=2-N^{+}=0$ coupling is slow, then only a fraction will be ionized in time for the ion to be detected. If the field is ramped up to that of a level crossing, then the wave function will develop a mixed $N^{+}=2 / N^{+}=0$ character over the fourth period of evolution during which the field is held constant, which could enhance the coupling to the continuum in the fifth phase. Before undergoing surface ionization, the wave function will undergo a further evolution due to the changing fields experienced as the molecule gets close enough to the surface to be affected by the image charges. Nevertheless, we suggest that these changing fields should not have a large effect on the extent of the $N^{+}=0$ admixture into the wave function, and hence in the final phase the system retains 

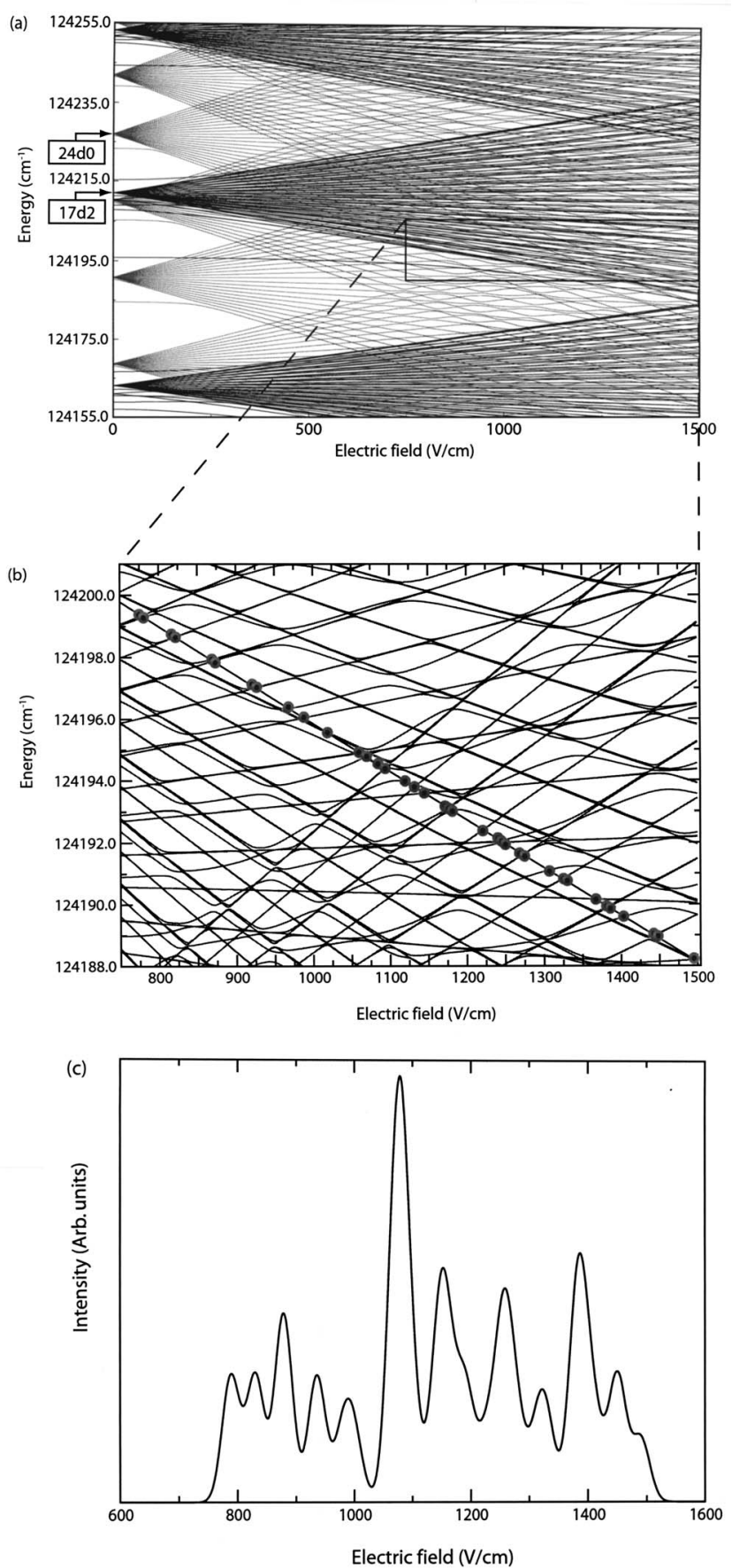

FIG. 10. (a) Stark map for $\mathrm{H}_{2}$ including all the $N^{+}=0(n=21-26)$ and $N^{+}$ $=2(n=16-18) M_{J}=0$ states (adapted from Ref. 12). The positions of the $N^{+}=0-N^{+}=2$ crossings for the state correlating adiabatically with the $(17 d 2)_{1}$ zero-field state are indicated in (b) by the circles as discussed in the text. In (c) a simulated surface ionization vs field profile is generated by summing a set of Gaussian peaks of equal height and width $\left(50 \mathrm{~V} \mathrm{~cm}^{-1}\right)$ at the positions of the level crossing marked in (b).

memory of its evolution in the fourth phase. It is clear that further theoretical and experimental work is desirable to fully evaluate the proposed resonant enhancement mechanism.

A similar but opposite argument can be presented to explain the presence of structure in the profiles recorded for the excitation of $N^{+}=0$ states. Rather than thinking of the structure as an enhancement of ionization probability leading to peaks in the profile, it is necessary to picture the structure as arising from dips in the profile associated with fields where the Rydberg state is less sensitive to ionization in the presence of the surface. Such a loss of sensitivity could arise from the $N^{+}=0$ state crossing back to an $N^{+}=2$ state (which requires a higher field for detection) at the crossing points.

The resonant behavior demonstrates that small changes in field can lead to significant changes in ionization probability, permitting a facile form of control over this physical process.

\section{F. Surface ionization behavior of states populated in a nonzero field}

In the presence of a strong electric field the electronic wave functions of a molecular Rydberg state mimic the parabolic Stark functions of the $\mathrm{H}$ atom in a field. ${ }^{7}$ The electron distribution is oriented such that the blueshifted states exhibit a wave function directed along the $+z$ axis, while the redshifted states have a wave function directed along the $-z$ axis, where the $z$ axis is defined as the field axis and is perpendicular to the surface plane. It is expected that these states will therefore show different surface ionization characteristics. In this work we have investigated this effect experimentally by selectively exciting members of the $N^{+}=2$ $n=18$ Stark manifold [whose intensity is derived from $(18 d 2)_{1}$ Rydberg states] in the presence of a $333 \mathrm{~V} \mathrm{~cm}^{-1}$ electric field. The Stark spectrum obtained at this field, with pulsed-field ionization detection, is presented in Fig. 11(a). Three different members of the manifold were chosen to characterize the range of ionization behavior exhibited by the $n=18$ Stark states. The chosen states are labeled in Fig. 11(a) and comprise (i) a redshifted state, (ii) a state in the center of the manifold, and (iii) a blueshifted state.

For each state an ion signal versus field profile was recorded in an analogous way to the previous investigations of zero-field states detailed above, and the results are presented in Fig. 11(b). Once again, the sudden drop in signal intensity at high field for each profile arises due to the molecules being field ionized in the gas phase following the application of the extraction pulse. The fact that excitation to blueshifted states results in the molecules being more readily ionized at lower fields can be explained by assuming that the states undergo field-induced rotational autoionization almost as soon as their energy is greater than the classical threshold for ionization to $N^{+}=0$. In Fig. 11(c) the lowering of the ionization limit for the $N^{+}=0$ channel as a function of applied field is superimposed on a Stark map of the $n=18$ energy levels for the $N^{+}=2$ states. The blueshifted states cross this threshold at lower field than the redshifted states; for the three states populated in the experiment it is estimated that the threshold for field-induced autoionization occurs at 650, 730, and $820 \mathrm{~V} \mathrm{~cm}^{-1}$, which is in each case approximately $50 \mathrm{~V} \mathrm{~cm}^{-1}$ below the observed onset of field ionization in Fig. 11, characterized by the start of the sharp drop in signal.

The surface-induced ionization behavior appears to show a similar trend; the main rise of the surface ionization signal in Fig. 11(b) (i) starts at around $600 \mathrm{~V} \mathrm{~cm}^{-1}$, whereas in Fig. 11(b) (iii) it starts at $400 \mathrm{~V} \mathrm{~cm}^{-1}$, implying that the blue- 


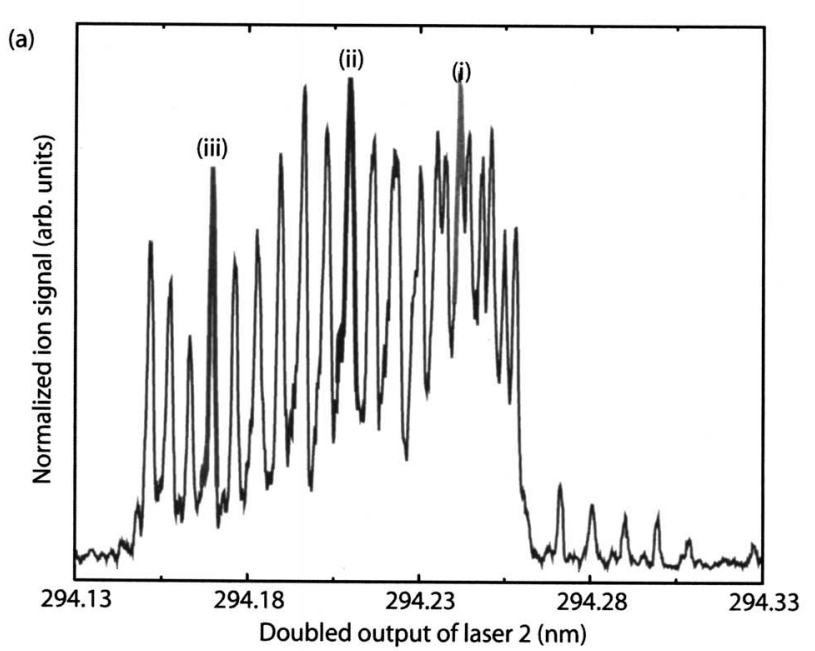

(b)

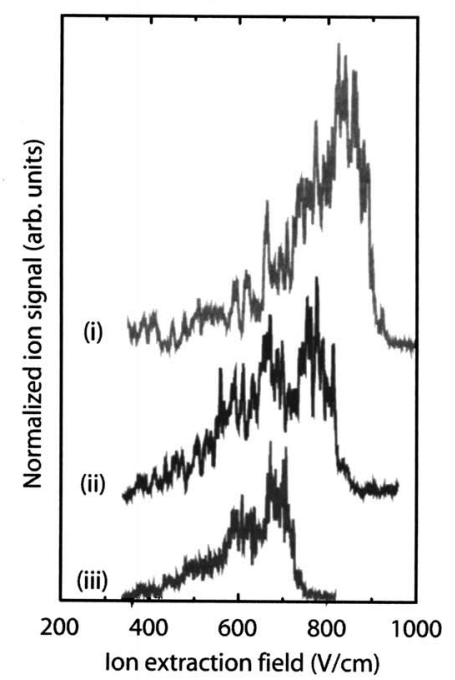

(c)

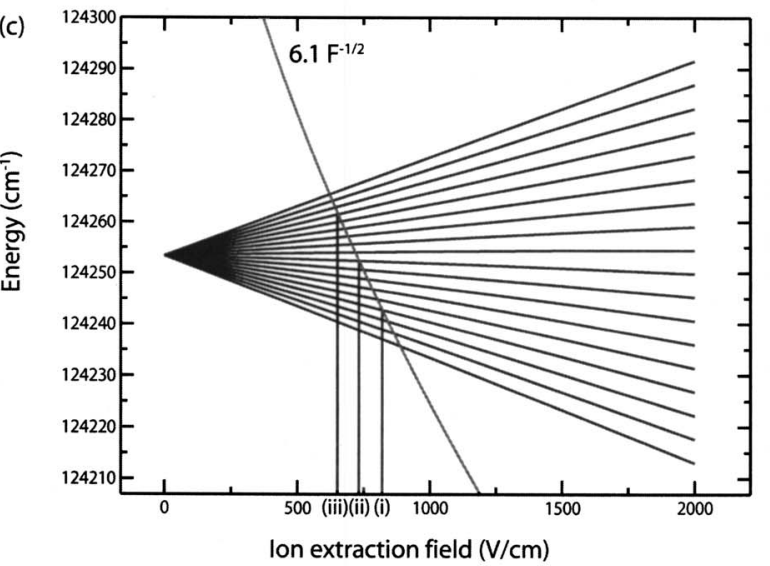

FIG. 11. (a) Stark spectrum of $\mathrm{H}_{2}$ for $N^{+}=2, n=18$, recorded with an applied field of $333 \mathrm{~V} \mathrm{~cm}^{-1}$ at the point of excitation and detected using pulsed-field ionization. (b) Surface ionization profiles corresponding to the population of the three states labeled (i)-(iii) in (a). (c) Stark map of the $n=18$ energy levels for $N^{+}=2$ (simulated using a simple linear-Stark-effect $\mathrm{H}$-atom model), with the field-lowered $N^{+}=0$ threshold indicated by the bold line.

shifted Stark states start to ionize at a greater distance from the surface. This behavior is different from that found in the previous experimental work with xenon atoms, ${ }^{22}$ where different Stark components show very similar surface ionization characteristics. The behavior is also the opposite from what might be expected intuitively in that the blueshifted Stark states (iii), which are ionized most easily at the greatest distance from the surface, are those whose wave function is oriented away from the surface towards the vacuum, whereas the redshifted states are those oriented towards the surface. Previous calculations of tunneling ionization rates for the $\mathrm{H}$ atom $^{22}$ suggest that the surface-oriented states should ionize at a greater distance from the surface and hence be extracted at lower field, contrary to the observation here.

In Ref. 27 the similar behavior of all the members of the Stark manifold for xenon Rydberg states was explained by considering the evolution of the state as the surface is approached. As the atom moves towards the surface, the field arising from the image charges induced in the metal increases, and the splitting between redshifted and blueshifted levels increases. Prior to reaching a sufficiently small atom/ surface separation for ionization to take place, level crossings with states in adjacent $n$ manifolds will occur. If these crossings are traversed adiabatically, then the character of a molecule initially in a state $\left|n k m_{l}\right\rangle$ will assume the character of the state $\left|n^{\prime} k^{\prime} m_{l}\right\rangle$. In general, this will correspond to a change between a redshifted and a blueshifted Stark state, and the polarized Rydberg electron wave function will jump from one orientation to the opposite orientation. As the surface is approached the system typically undergoes many such avoided crossings, and the overall effect of this is that the electron probability densities associated with the blueshifted and redshifted states initially populated will, on average, be similar. Consequently, the surface ionization behavior of the blueshifted and redshifted Stark states is expected to be the same. A recent theoretical paper ${ }^{28}$ has suggested that it is the externally applied ion-extraction field which brings about the level crossings, the extraction field being already large enough to take the system through the Inglis-Teller limit where adjacent $n$ manifolds cross.

In the hydrogen molecule, however, the situation is potentially different for the $N^{+}=2$ states investigated: the primary mechanism for surface ionization involves surfaceinduced rotational autoionization, as described above, and for the $N^{+}=2$ states the ionization occurs at much greater distance from the surface than for the same principal quantum number in xenon. For the states investigated here, it appears that the surface ionization occurs before the system has passed through any level crossings with adjacent $N^{+}=2$ Stark manifolds $\left(n^{\prime}=n \pm 1\right)$.

The explanation for the observed greater propensity for the blueshifted Stark states to ionize is similar to that describing the field-induced autoionization in the gas phase. As the molecule approaches the surface, the threshold of the $N^{+}=0$ continuum is lowered by the surface potential; surface-induced autoionization starts to occur as soon as the energy of the populated $N^{+}=2$ state (shifted by the external field and the surface interaction) lies above the surface-fieldlowered $N^{+}=0$ threshold. In this way we expect the blueshifted Stark states to ionize at a greater distance from the surface, as is observed. 


\section{CONCLUSIONS}

In this work we have demonstrated that the behavior of a molecular Rydberg state interacting with a metal surface can be significantly different from an atomic system. The experimental results suggest that, when close to the metal surface, the Rydberg molecules undergo a process of surface-induced rotational autoionization; i.e., the rotational energy of the $\mathrm{H}_{2}^{+}$ ion core of the Rydberg state contributes to the ionization process, such that the ionization occurs at a greater distance from the surface than occurs with atomic Rydberg states of the same $n$. Remarkably, it was also found that the surface ionization cross section shows strong resonances as a function of the applied electric field, which were independent of the metal studied. The resonant fields correspond to crossings between energy levels of different rotational quantum number; and these findings imply that a degree of control can be exerted over the rate of ionization by a small adjustment of field. It was found that, contrary to the behavior observed in atomic systems, individual Stark states undergo surface ionization without passing through level crossings with adjacent Stark manifolds of the same core rotational quantum number, and therefore the ionization behavior is dependent on which level of the Stark manifold is initially populated.

One of the key conclusions from this work is that the surface ionization is very much like the field ionization in the gas phase; in the former case the field is created by the image charges in the metal, whereas in the latter case it is externally applied. The question is just how similar are these two processes? In fact, there are two significant physical differences that emerge from a detailed theoretical treatment. The first is that in the surface ionization process, the electron must escape from the molecule/atom in the direction of the surface, and in this direction its potential energy does not tend towards $-\infty$, as in the gas-phase process, but must join smoothly on to the potential within the bulk metal. The second difference is that although the field created by the image charges is cylindrically symmetric on average about the normal to the surface, it is actually very strongly inhomogeneous across the dimensions of the Rydberg orbit at the typical distance at which ionization occurs. In fact, the average field experienced by the Rydberg electron at a given distance from the surface can only be determined through a knowledge of the electronic eigenfunctions. The combined impact of both these factors is illustrated in calculations of the energy levels of a $\mathrm{H}$ atom as a function of distance from the surface, as shown, for example, in Fig. 2 of Ref. 37. Thus, the energy levels do not simply spread out monotonically as the surface is approached (and the average field increases) but show a more complex behavior. Moreover, the eigenfunctions are not simply the parabolic Stark functions of the $\mathrm{H}$ atom, as would be the case in a homogeneous field. These factors may have a significant influence on the surface ionization rate for a given initially populated level and distance from the surface. Thus, the analogy with gas-phase field ionization is helpful in a qualitative discussion, but has a limited value in a quantitative treatment.

The use of Rydberg molecules, such as $\mathrm{H}_{2}$ in surfacescattering experiments, presents several other possible new aspects to the physical problem, compared to electronically excited atoms, which remain to be investigated. First, the molecular ion core can possess not only rotational degrees of freedom but also vibrational degrees of freedom, and therefore the question arises as to how these might contribute to the interaction with the surface and the tunneling ionization probability. Second, the Rydberg molecule has sufficient energy to undergo dissociation into neutral species (e.g., $\mathrm{H}_{2}^{*}$ $\rightarrow \mathrm{H}+\mathrm{H}$ ), and therefore the collision with the surface could induce predissociation in competition with ionization. There is also the possibility of other types of chemical process occurring: The products of tunneling ionization $\left(\mathrm{H}_{2}^{+}\right)$or dissociation ( $\mathrm{H}$ atoms) could potentially become involved in secondary chemical processes at the surface. Alternatively, the interaction of a Rydberg molecule with an adsorbate could proceed via the transfer of the Rydberg electronic energy into the breaking of a chemical bond in the adsorbate, followed by secondary chemistry, possibly involving reaction with the deexcited incident molecule. To date none of these processes have been studied experimentally for Rydberg-surface collisions.

In a broader context there is a growing interest in the interactions of Rydberg atoms and molecules with a variety of types of surface. An area of great technological importance is the use of plasmas for film deposition and etching, and plasma-surface interactions lie at the heart of such processes. It has been established that in argon plasmas, metastable electronically excited atoms at the surface-plasma boundary play a crucial role in determining the characteristics of the plasma. ${ }^{38}$ Long-lived Rydberg species are likely to be abundant in many types of plasma ${ }^{39}$ and will make important contributions to the chemistry, ${ }^{40}$ but the role of Rydberg-surface interactions in plasmas is not known, and the study of Rydberg-surface interactions will provide important new information for modeling such media. Charge exchange at surfaces is an important phenomenon in practical applications ranging from catalytic processes to high-energy ion sputtering to nanoscale deposition. Ion sputtering is known to proceed via the formation of hollow atoms with many electrons in high Rydberg orbitals; the Rydberg electrons are stripped off as the ion approaches the surface. ${ }^{41}$ Rydberg atoms and molecules provide a controllable model system for studying the fundamentals of charge exchange processes. It has also been proposed that Rydberg atoms could be used for nanoscale pattern deposition on surfaces, ${ }^{42}$ especially as the spatial distribution of the Rydberg atoms could be easily controlled using low-intensity standing wave laser fields ${ }^{42}$ or inhomogeneous electric field focusing. ${ }^{15,16}$ There is a close connection between the work proposed here and experiments/theory studying electronically excited states of surfaces and of surface-bound species for both nonmetals and metals. There is experimental evidence for the thermal emission of Rydberg species (atoms, molecules, and clusters) from impregnated metal oxide surfaces ${ }^{43-45}$ thus, studies such as those initiated in the present work may help to understand these desorption processes through the applicability of microscopic reversibility. The Rydberg species are thought to originate from the presence of electronically excited species at the surface. ${ }^{43,44}$ Femtosecond laser studies of elec- 
tronically excited states at metallic surfaces have revealed the existence of Rydberg-like image potential states of electrons; ${ }^{46}$ the theoretical description of these is closely linked to the image charge potential described above. There has also been a recent claim that empty Rydberg orbitals in saturated hydrocarbons can play an important role in adsorbate-surface bonding. ${ }^{47}$

In the area of chemical dynamics, the interaction of Rydberg molecules with surfaces could offer new opportunities for studying and controlling reactive processes at a surface. The possibility to control not only the electronic energy and spatial properties of the electronic wave function, but also the spatial divergence and the velocity of impact of the Rydberg beams ${ }^{11,12,15}$ would be a valuable feature of such experiments.

The work presented in this paper points to the possibility of opening a new field of applications and fundamental science for investigation.

\section{ACKNOWLEDGMENTS}

The authors are grateful to the EPSRC for financial support of this work, and to Dr. R. M. J. Jacobs for his assistance with the preparation and characterization of the surface samples.

${ }^{1}$ G. O. Sitz, Rep. Prog. Phys. 65, 1165 (2002).

${ }^{2}$ L. Pasquali and S. Nannarone, Nucl. Instrum. Methods Phys. Res. B 230, 340 (2005).

${ }^{3}$ S. J. H. Petra, L. Feenstra, W. Hogervorst, and W. Vassen, Appl. Phys. B: Lasers Opt. B78, 133 (2004).

${ }^{4}$ D. C. Jordan, I. S. T. Tsong, D. J. Smith, B. J. Wilkens, and R. B. Doak, Appl. Phys. Lett. 77, 3030 (2000).

${ }^{5}$ R. T. Jongma, G. Berden, D. van der Zande, T. Raising, H. Zacharias, and G. Meijer, Phys. Rev. Lett. 78, 1375 (1997).

${ }^{6}$ G. R. Lloyd, S. R. Procter, and T. P. Softley, Phys. Rev. Lett. 95, 133202 (2005).

${ }^{7}$ T. P. Softley, Int. Rev. Phys. Chem. 23, 1 (2004).

${ }^{8}$ S. R. Procter, M. J. Webb, and T. P. Softley, Faraday Discuss. 115, 277 (2000).

${ }^{9}$ T. P. Softley, A. J. Hudson, and R. Watson, J. Chem. Phys. 106, 1041 (1997).

${ }^{10}$ D. Townsend, A. L. Goodgame, S. R. Procter, S. R. Mackenzie, and T. P. Softley, J. Phys. B 34, 439 (2001).

${ }^{11}$ S. R. Procter, Y. Yamakita, F. Merkt, and T. P. Softley, Chem. Phys. Lett. 374, 667 (2003).

${ }^{12}$ Y. Yamakita, S. R. Procter, A. L. Goodgame, T. P. Softley, and F. Merkt, J. Chem. Phys. 121, 1419 (2004).
${ }^{13}$ E. Vliegen, H. J. Woerner, T. P. Softley, and F. Merkt, Phys. Rev. Lett. 92, 033005 (2004).

${ }^{14}$ E. Vliegen and F. Merkt, J. Phys. B 38, 1623 (2005); 39, L241 (2006).

${ }^{15}$ O. Kritsun and H. Metcalf, Bull. Am. Phys. Soc. 46, 109 (2002).

${ }^{16}$ E. Vliegen and F. Merkt, Phys. Rev. Lett. 97, 033002 (2006).

${ }^{17}$ S. A. Deutscher, X. Yang, and B. Joachim, Phys. Rev. A 55, 466 (1977).

${ }^{18}$ S. B. Hill, C. B. Haich, Z. Zhou, P. Nordlander, and F. B. Dunning, Phys. Rev. Lett. 85, 5444 (2000).

${ }^{19}$ C. A. Kocher and C. R. Taylor, Phys. Lett. A 124, 68 (1987).

${ }^{20}$ G. E. McCown, C. R. Taylor, and C. A. Kocher, Phys. Rev. A 38, 3918 (1988).

${ }^{21}$ P. Nordlander and J. C. Tully, Surf. Sci. 211-212, 207 (1989).

${ }^{22}$ P. Nordlander, Phys. Rev. B 53, 4125 (1996).

${ }^{23}$ C. Fabre, M. Gross, J. M. Raimond, and S. Haroche, J. Phys. B 16, L671 (1983).

${ }^{24}$ K. Ganesan and K. T. Taylor, J. Phys. B 29, 1293 (1996).

${ }^{25}$ U. Thumm, P. Kurpick, and U. Wille, Phys. Rev. B 61, 3067 (2000).

${ }^{26}$ F. B. Dunning, H. R. Dunham, C. Oubre, and P. Nordlander, Nucl. Instrum. Methods Phys. Res. B 203, 69 (2003).

${ }^{27}$ S. Wethekam, H. R. Dunham, J. C. Lancaster, and F. B. Dunning, Phys. Rev. A 73, 032903 (2006).

${ }^{28}$ J. Sjakste, A. G. Borisov, and J. P. Gauacq, Phys. Rev. A 73, 042903 (2006).

${ }^{29}$ A. Anderson, S. Haroche, E. A. Hinds, W. Jhe, and D. Meschede, Phys. Rev. A 37, 3594 (1988).

${ }^{30}$ R. Bruhl, P. Fouquet, R. E. Grisenti, J. P. Toennies, G. C. Hegerfeldt, T. Kohle, M. Stoll, and C. R. Walter, Europhys. Lett. 59, 357 (2002).

${ }^{31}$ J. Mozley, P. Hyafil, G. Nogues, M. Brune, J.-M. Raimond, and S. Haroche, Eur. Phys. J. D 35, 43 (2005).

${ }^{32}$ A. A. Radzig and B. M. Smirnov, Reference Data on Atoms, Molecules, and Ions (Springer, New York, 1985), p. 396.

${ }^{33}$ J. M. Gilligan and E. E. Eyler, Phys. Rev. A 46, 3676 (1992).

${ }^{34}$ S. R. Mackenzie and T. P. Softley, J. Chem. Phys. 101, 10609 (1994).

${ }^{35}$ A. L. Goodgame, H. Dickinson, S. R. Mackenzie, and T. P. Softley, J. Chem. Phys. 116, 4922 (2002).

${ }^{36}$ T. J. Morgan, C. F. Barnett, J. A. Ray, and A. Russek, Phys. Rev. A 20, 1062 (1979).

${ }^{37}$ F. Nordlander and F. B. Dunning, Phys. Rev. B 53, 8083 (1996).

${ }^{38}$ T. Sakurai and M. Toda, Thin Solid Films 374, 157 (2000).

${ }^{39}$ R. S. Mason, P. D. Miller, I. Mortimer, D. J. Mitchell, and N. A. Dash, Phys. Rev. A 68, 016408 (2003).

${ }^{40}$ K. Hassouni, A. Gicquel, and M. Capitelli, Chem. Phys. Lett. 290, 502 (1998).

${ }^{41}$ N. Stolterfoht, D. Niemann, M. Grether, A. Spieler, and A. Arnau, Scanning Microsc. 12, 437 (1998).

${ }^{42}$ N. A. Nguyen, B. K. Dey, M. Shapiro, and P. Brumer, J. Phys. Chem. A 108, 7878 (2004)

${ }^{43}$ L. Holmlid, J. Phys. Chem. A 102, 10636 (1998).

${ }^{44}$ L. Holmlid, J. Phys.: Condens. Matter 14, 13469 (2002).

${ }^{45}$ J. Wang and L. Holmlid, Chem. Phys. 261, 481 (2000).

${ }^{46}$ U. Hofer, I. L. Shumay, Ch. Reuss, U. Yhomann, W. Wallauer, and Th. Fauster, Science 277, 1480 (1997).

${ }^{47}$ K. A. Fosser, R. G. Nuzzo, P. S. Bagus, and C. Woll, Angew. Chem., Int. Ed. 41, 1735 (2002). 